\title{
Sexually transmitted infections among transgender people and men who have sex with men in Port Vila, Vanuatu
}

\author{
Vanessa Veronese, ${ }^{a}$ Caroline van Gemert, ${ }^{a}$ Siula Bulu, ${ }^{b}$ Tamara Kwarteng, ${ }^{c}$ Isabel Bergeri, ${ }^{a}$ Steven Badman, ${ }^{d}$ \\ Alyce Vella and Mark Stoovéae \\ Correspondence to Mark Stoové (e-mail: stoove@burnet.edu.au).
}

Despite high sexually transmitted infection (STI) prevalence in the Pacific, there are limited data on STIs and risk among men who have sex with men (MSM) and transgender people (TG). In 2011, an Integrated Bio-Behavioural Survey recruited self-identified MSM and TG in Port Vila, Vanuatu. Descriptive findings were stratified by sexuality. Among 28 (55\%) MSM and 23 (45\%) TG, recent anal sex with male partners was more common among MSM (94\% vs $71 \% ; P<0.1)$, including with casual ( $47 \%$ vs $35 \%$ ), regular ( $59 \%$ vs $29 \%$ ) and paying partners ( $28 \%$ vs $12 \%$ ). MSM more commonly reported lifetime $(P<0.01)$ and recent sex with female partners $(P<0.01)$. Reported condom use with any partner type was low. More MSM (35\%) than TG (24\%) were diagnosed with an STI; previous treatment-seeking behaviour when symptomatic was lower among TG $(P<0.1)$. Tailored strategies acknowledging differences between MSM and TG are required to reduce STI vulnerability in Vanuatu.

$\mathrm{D}$ espite a high prevalence of sexually transmitted infections (STIs) in Pacific island countries and territories, ${ }^{1-4}$ there are limited data on STIs and risk behaviour among men who have sex with men (MSM) and transgender people (TG). While culturally defined TG roles for men exist throughout Pacific island countries and territories, ${ }^{5}$ male-to-male sexual contact occurs among men who do not identify as TG or homosexual. In Vanuatu, up to $10 \%$ of young males reported sex with a male partner. ${ }^{6}$ Stigma and discrimination towards sexual minorities deters disclosure of sexuality and male-tomale sexual behaviour. This can result in low levels of STI testing and diagnosis, underreporting of risk behaviours and limited engagement with prevention and treatment services. $^{5,7-9}$

The limited data for MSM and TG populations in Pacific island countries and territories hamper risk reduction among these groups and their sexual partners. In 2011, an Integrated Bio-Behavioural Survey of selfidentified MSM and TG was conducted by the Burnet Institute in partnership with Wan Smolbag and the Vanuatu Ministry of Public Health in Port Vila, Vanuatu.

\section{METHODS}

\section{Recruitment and data collection}

Respondent-driven sampling (RDS) was used to recruit participants between November 2011 and April 2012. Biological men, identifying as male or transgender women, and aged over 18 years who reported anal sex with another male in previous year were eligible.

Seven seeds (initial recruits) were recruited through a local nongovernmental organization and chosen for their close linkages with the study population and for diversity (e.g. gender, age, marital status). Seeds were given three coupons to recruit the first wave of participants who were also provided three coupons to recruit the next wave, and so on; willing participants then presented for data collection.

Three field researchers assessed eligibility and obtained informed verbal consent. Interviews were conducted in the local language; the questionnaire included items about socio-demographics, sexual risk

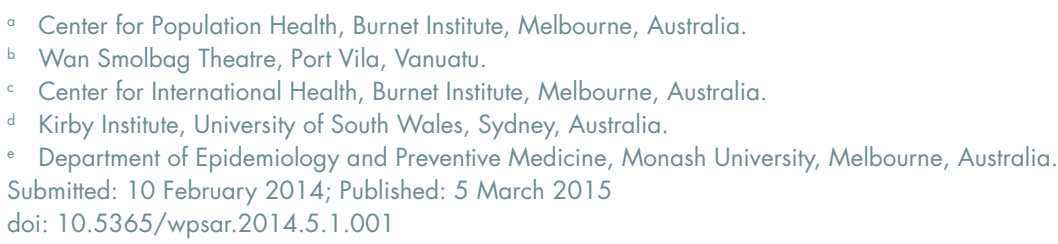


behaviours, previous diagnoses of any STI, prior STI symptoms and treatment-seeking behaviour. Participants were then referred for pre-test counselling and STI testing. Nurses collected 20 millilitres of venous blood for HIV and syphilis testing and rectal swabs for chlamydia and gonorrhoea testing. Chlamydia and gonorrhoea tests were performed using BD ProbeTec (Becton, Dickinson and Company, New Jersey, USA) nucleic acid amplification testing; tests for active syphilis used RPR screening confirmed through Determine ${ }^{\mathrm{TM}}$ Syphilis TP (Inverness Medical, Bedford, UK). For HIV, a rapid screening test (Determine ${ }^{\mathrm{TM}}$ ) was used with confirmation of reactive samples by two additional rapid tests (Insti, BioLytical, British Columbia, Canada and Unigold, Trinity Biotech, Carlsbad, USA).

\section{Analysis}

Most variables were dichotomous or categorical; these were analysed using Fisher's exact test. For numerical variables, the median and interquartile ranges were calculated. Due to the small sample size and limited recruitment, the data could not be analysed using methods for RDS. Analyses were conducted using Epilnfo and StataSE v11.

To determine recent STI exposure, participants were asked if they had experienced any of four STI symptoms in the previous 12 months: (1) genital discharge from the vagina; (2) genital ulcer or sore on the vagina; (3) burning or sharp pain on urination; and (4) rash or genital itching. Participants were then classified as self-reporting symptoms or not during this time period. Consistent condom use was defined as always using a condom with partner during the previous six months.

The Ministry of Health, Government of the Republic of Vanuatu Ethics Committee and the Alfred Hospital Ethics Committee approved this study. No identifying information was collected from participants.

\section{RESULTS}

Six seeds recruited 45 participants (total $n=51$ ); $23(45 \%)$ identified as TG and 28 (55\%) as MSM. An additional 20 MSM refused to participate, citing fear of disclosure of sexuality or an unwillingness to test for HIV and STIs. The most common age group was $20-29$ years; $21 \%$ of MSM and $17 \%$ of TG were aged 19 years or under.

TG participants reported younger age at sexual debut compared to MSM (16 or younger $(P<0.05$ ). Condom use at first sex was low among all respondents. While a history of forced sex was high overall (47\%), it was more commonly reported among TG $(P<0.1)$ (Table 1).

The majority of participants reported sexual contact with a male partner during the past six months. Consistent condom use during anal sex was low across all regular, casual, paying and paid sexual partners (Table 1). We also found evidence of multiple and potentially overlapping sexual partnerships; among the eight MSM reporting regular partners in the past six months, three also reported paid partners, three reported casual partners, one reported a paying partner and four reported female partners.

Significantly more MSM reported sex with a female partner compared to TG $(P<0.01)$; the majority of MSM reported sex with a female during the past six months. Consistent condom use with recent female partners was low overall (Table 1).

Approximately one in five MSM and no TG had a history of STI diagnosis $(P=0.03)$, and one third of MSM and one in five TG reported genital symptoms in the past year. MSM reported higher levels of genital symptoms across all four types investigated and were also significantly more likely to seek treatment for these symptoms $(P=0.02)$ (Table 1 ).

For the 43 participants that provided a sample, $24 \%$ of TG and $35 \%$ of MSM tested positive for any STI; chlamydia was detected in $19 \%$ of samples and gonorrhoea in $9 \%$. Of the 39 participants tested for syphilis, $3 \%$ were positive (Table 1 ). There were no detected cases of HIV.

\section{DISCUSSION}

While acknowledging the limitations in our sample size, these findings nonetheless advance our understanding 
Table 1. Socio-demographic and self-reported sexual risk and STI exposure and treatment history by reported gender $(n=51)$

\begin{tabular}{|c|c|c|c|}
\hline & TG $(n=23)$ & $\operatorname{MSM}(n=28)$ & $p$-value \\
\hline Socio-demographic & n (\%) & n (\%) & \\
\hline Age group (years) & & & 0.235 \\
\hline 19 and below & $4(17)$ & $6(21)$ & \\
\hline $20-29$ & 9 (39) & $16(57)$ & \\
\hline 30 and above & $10(43)$ & $6(21)$ & \\
\hline Median age (range) & $26(18-48)$ & $25(18-46)$ & \\
\hline \multicolumn{4}{|l|}{ Sexual risk } \\
\hline Age at first sex & & & 0.03 \\
\hline 16 and below & $17(74)$ & $10(36)$ & \\
\hline $16-19$ & $4(17)$ & $12(43)$ & \\
\hline 20 and above & $2(9)$ & $6(21)$ & \\
\hline Median age at first sex (range) & $12(7-22)$ & $16(12-28)$ & \\
\hline Condom use at first sex & $2(9)$ & $4(14)$ & - \\
\hline Forced sex ever & $14(61)$ & $10(36)$ & 0.07 \\
\hline \multicolumn{4}{|l|}{ Male partners } \\
\hline Any sexual contact with male partner (past six months) & $17(74)$ & $18(64)$ & \\
\hline Anal sex & $12(71)$ & $17(94)$ & 0.06 \\
\hline Oral sex & $17(100)$ & $13(72)$ & 0.02 \\
\hline Median number of partners (range) & $2(0-12)$ & $4(0-21)$ & \\
\hline Regular partner & $6(35)$ & $8(44)$ & \\
\hline Median number of regular partners (range) & $3(2-8)$ & $3(1-6)$ & \\
\hline Consistent condom use with regular partner & 0 & $1(12)$ & \\
\hline Casual partner & $5(29)$ & $10(56)$ & \\
\hline Median number of casual partners (range) & $7(2-20)$ & $2(1-4)$ & \\
\hline Consistent condom use with casual partner & 0 & 0 & \\
\hline Received payment for sex & $2(12)$ & $5(28)$ & \\
\hline Median number of casual partners (range) & $2(1-2)$ & $1(1-2)$ & \\
\hline Condom use at last sex with paying partner & 0 & $3(60)$ & \\
\hline Paid for sex last 12 months & 1 & 3 & \\
\hline Median number of paid partners (range) & 1 & $2(1-4)$ & \\
\hline Condom use at last sex with paid partners & 0 & 0 & \\
\hline \multicolumn{4}{|l|}{ Female partners } \\
\hline Female sex ever & $7(30)$ & $24(86)$ & $<0.01$ \\
\hline Female sex last six months & $5(71)$ & $21(88)$ & 0.31 \\
\hline Median number of female partners (range) & $1(0-3)$ & $2(0-11)$ & \\
\hline Consistent condom use with female partner & $1(20)$ & $1(5)$ & \\
\hline \multicolumn{4}{|l|}{ STI exposure and treatment seeking history } \\
\hline Ever diagnosed with a STI & 0 & $5(18)$ & 0.03 \\
\hline Genital symptoms past 12 months & $4(17)$ & $10(36)$ & \\
\hline Sought treatment for these symptoms & 0 & $7(70)$ & 0.02 \\
\hline at $\mathrm{KPH}$ clinic & 0 & $5(71)$ & \\
\hline at hospital & 0 & $1(14)$ & \\
\hline at other location & 0 & $1(14)$ & \\
\hline STI diagnosis & TG $(n=19)$ & MSM $(n=24)$ & \\
\hline Chlamydia & $3(16)$ & $5(21)$ & \\
\hline Gonorrhoea & $2(11)$ & $2(8)$ & \\
\hline Syphilis* & 0 & $1(5)$ & \\
\hline HIV & 0 & 0 & \\
\hline Any STI diagnosed ${ }^{\dagger}$ & $4(24)$ & $7(35)$ & \\
\hline
\end{tabular}

MSM, men who have sex with men; STI, sexually transmitted intection; TG, transgender.

* Denominator TG $=18 ; \mathrm{MSM}=21$

${ }^{\dagger}$ Denominator $\mathrm{TG}=17 ; \mathrm{MSM}=20$ 
of the sexual health vulnerabilities for MSM and TG in Vanuatu, a region characterized by distinctive and culturally defined sexualities.

The detection of STIs in one in five TG and one in three MSM represents comparably high prevalence for Pacific island countries and territories., ${ }^{1,3}$ No TG and only five MSM reported an STI diagnosis history. That $17 \%$ of TG and $36 \%$ of MSM reported STI symptoms in the past 12 months suggests low treatment-seeking behaviour, particularly among symptomatic TG and may reflect barriers to accessing treatment. In settings where same-sex behaviour is not culturally permissible, TG may be unwilling to access mainstream sexual health services where they might be asked to disclose sexual behaviours. ${ }^{10}$ However, services specifically targeting gay men may not appeal to bisexual MSM who maintain relationships with women and thus present a barrier to care and preventative treatment among this group. Our findings underscore the needs for culturally appropriate health promotion and sexual health services that reflect the specific needs of MSM and TG populations.

We found consistently low levels of condom use with regular and casual partners. Lifetime and recent sex with females by MSM was also higher than reported elsewhere. ${ }^{1,9}$ Against a background of high STI prevalence, low levels of condom use with female partners, high levels of bisexual risk behaviours and multiple and potentially overlapping male and female sexual partnerships create a potential bridge between high-risk groups and the general population. It is crucial that approaches to preventing and treating STIs in Vanuatu incorporate discrete strategies for partner notification and treatment for female partners.

This study was subject to limitations which must be considered when reviewing the findings. Our small sample size limited the detection of particular outcomes and statistical power that may have been observed in a larger sample. The RDS strategy employed also did not result in sufficient recruitment. Our question about participant's age at sexual debut implied but did not specify homosexual sex; thus, our findings may not accurately represent the age of homosexual debut among participants.

Despite this, our results show important risk behaviours and profiles of TG and MSM in Vanuatu not previously documented. Overall, high and somewhat specific levels of risk and vulnerability among TG and MSM in Vanuatu were determined. Our findings underscore the need for comprehensive and targeted STI treatment and prevention services for MSM and tailored health promotion strategies that recognizes different sexual identities and adequately engages non-gay identifying MSM and their female partners in Vanuatu.

\section{Conflicts of interests}

None declared.

\section{Funding}

Funding was provided by the Pacific Island HIV and STI Response Fund grant, a collaborative funding mechanism by the Australia and New Zealand governments and managed by the Secretariat of the Pacific Community.

\section{Acknowledgements}

We wish to thank the Vanuatu Ministry of Health for its ongoing support and for laboratory testing; National Serology Reference Laboratory (NRL, Melbourne, Australia) for laboratory testing; Wan Smolbag for coordinating data collection and entry and management of the field researchers; WHO Western Pacific Regional Office and the Pacific UNAIDS Coordinator for their technical support in project implementation. Caroline van Gemert received a Travel Grant from the lan Potter Foundation for training in RDS. Laboratory testing of specimens at the Port Vila Central Hospital was provided by the Global Fund Round 7 Grant through the Vanuatu Ministry of Health. Finally, we thank all participants of the study for their valuable input. We would also like to thank William Craw for his valuable advice.

\section{References:}

1. Rawstorne P et al. Shattered Stereotypes: Twists \& Turns in Sexual Practices. An integrated bio-behavioural survey of transgender and men who have sex with men in Suva and Lautoka, Fiji. Suva, MEN Fiji, 2012.

2. Rawstorne P, Te Tiare Association of the Cook Islands, Secretariat of the Pacific Community. Second Generation Surveillance Survey of Akavaine and MSM in Cook Islands 2009. Cook Islands, Secretariat of the Pacific Community, 2009.

3. Second Generation Surveillance Survey of MSM in Tonga 2008. Tonga, Ministry of Health, 2008.

4. Second Generation Surveillance: Behavioural survey among MSM. Guam, Guam Department of Public Health and Social Services, 2007. 
5. Buchanan-Aruwafu H. An Integrated Picture: HIV risk and vulnerability in the Pacific - Research Gaps, Priorities and Approaches. New Caledonia, Secretariat of the Pacific Community, 2007.

6. I No bin gat protection. Understanding HIV and AIDS risk and vulnerability among Vanuatu Youth. Suva, United Nations Internation Children's Fund Pacific Sub-Regional Office, 2010.

7. HIV Surveillance in Pacific Island Countries and Territories: 2012 report. New Caledonia, Surveillance and Operational Health Research Team, Secretariat of the Pacific Community, 2013.
8. Godwin J. Legal environments, human rights and HIV responses among men who have sex with men and transgender people in Asia and the Pacific: An agenda for action. Bangkok, United Nations Development Programme, 2010.

9. Bavington B et al. Secret Lives, Other Voices... A communitybased study exploring male-to-male sex, gender identity and HIV transmission risk in Fiji. Suva, AIDS Task Force of Fiji, 2011.

10. Sullivan PS et al. Successes and challenges of HIV prevention in men who have sex with men. Lancet, 2012, 380:388-389. doi:10.1016/S0140-6736(12)60955-6 pmid:22819659 